curdy material. The effect of this treatment was remarkable. In a few days the ulcers cleaned and the purulent discharge diminished in quantity. The process of repair in the ulcerated surfaces was very rapid and after two months' persistent local disinfection the back, and after four months the whole of the areas involved (back, arm, legs) had completely healed. It is not necessary to give illustrations of the present condition as all they would show is extensive cicatrisation.

At the time of writing the patient is in excellent health. He is able to attend to business and his weight has increased to 11 stones. I thought that owing to contraction of cicatricial tissue walking would prove a difficulty and that it would be necessary to do something in order to free the action of the knee-joints. However, no further treatment of this kind has been required as their movement is quite free.

In conclusion, it may be mentioned that it was the opinion of the late Mr. Jessop, when he operated, that the case was tuberculous in origin.

Harrogate.

\section{THE OPERATION OF JEJUNOSTOMY,} WITH A DESCRIPTION OF A NEW METHOD. ${ }^{1}$

By A. W. MAYO ROBSON, D.Sc. LEEDS, F.R.C.S. ENG., SENIOR VICE-PRESIDENT OF THE ROYAL COLLEGE OF SURGEONS OF ENGLAND.

JeJurostomy is an operation occasionally called for as a means of giving relief and prolonging life in patients suffering: from advanced disease of the stomach, where on exploration it is discovered to be impracticable to perform gastrectomy, gastrostomy, or gastro-enterostomy. The indications for the operation are (1) extensive cancer of the stomach too advanced for gastrectomy and in which no healthy "spot of sufficient size on the stomach wall can be found for the purpose of gastrostomy or gastro-enterostomy ; and (2) general cicatricial contraction of the stomach, simple in character and due to the swallowing of caustic fluid, in which the stomach has been so far damaged that it no longer performs its functions or even allows of the proper passage onwards of food. It has also been suggested in pronounced hyperchlorhydria in preference to gastro-enterostomy in order to avoid peptic ulcer of the jejunum, but as the latter is extremely rare and practically only associated with anterior gastro-enterostcmy, a method that is being replaced by the posterior operation, I do not think surgeons generally will be likely to endorse Neumann's suggestions; nor do I think that it is probable that jejunostomy will be likely to replace other methods of securing rest in the treatment of gastric ulcer or of hæmatemesis, as Cackovie has suggested, and as has been adopted on one occasion with success by Mr. Willoughby Furner.

Modes of operation.-For any operation to be a success the bowel must be so placed that it will serve the two purposes: (1) to permit the passage onward of the bile and pancreatic fluid poured into the intestine above the artificial fistula; and (2) to allow of food being introduced through the fistula without fear of regurgitation, either of the food or of the intestinal contents. In my first operation, performed in 1891, I used a method which, though successful in prolonging life, was inconvenient and cumbersome and I need not mention it further.

The operations now used are the following:-1. A modification of Witzel's method of gastrostomy in which a No. 12 rubber catheter is stitched into an opening in the jejunum and afterwards the catheter is buried in a groove in the bowel for a distance of about two inches, the line of suture being fixed to the abdominal wall (see Figs. 1 and 2). 2. Maydl's method of dividing the jejunum, implanting the proximal cut end into the distal portion a few inches from the original line of section, the open end of this section being fixed to the skin (see Fig. 3). 3. The operation which I have performed and which I believe is new. It consists in taking a loop of the beginning of the jejunum just sufficiently long to reach the surface without tension; the two arms of the loop are short-circuited about three or four

1 A paper read before the Royal Medical and Chirurgical Society on April 11th, 1905. inches from the surface, the short-circuiting being done either by means of sutures around a decalcified bone bobbin or by sutures alone; personally I prefer the former. A small incision is then made into the top of the loop just large enough to admit a No. 12 Jaques catheter, which is Fig. 1.

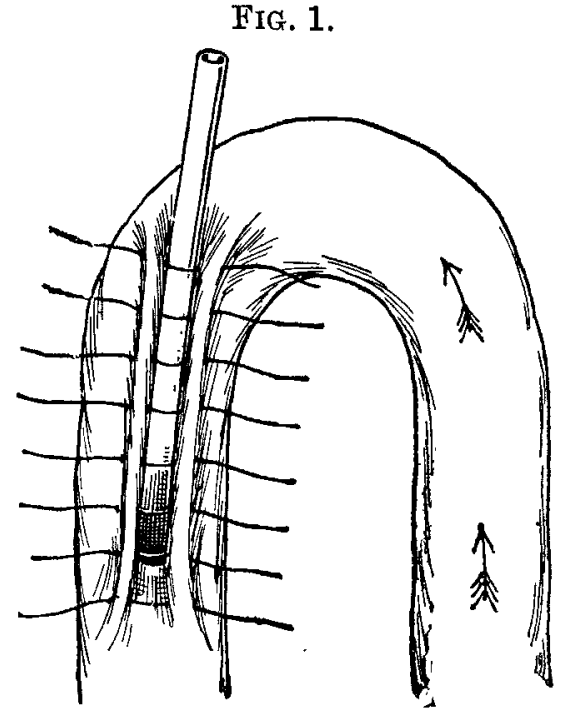

A method of performing jejunostomy by a modification of Witzel's operation for gastrostomy. First stage.

FIG. 2.

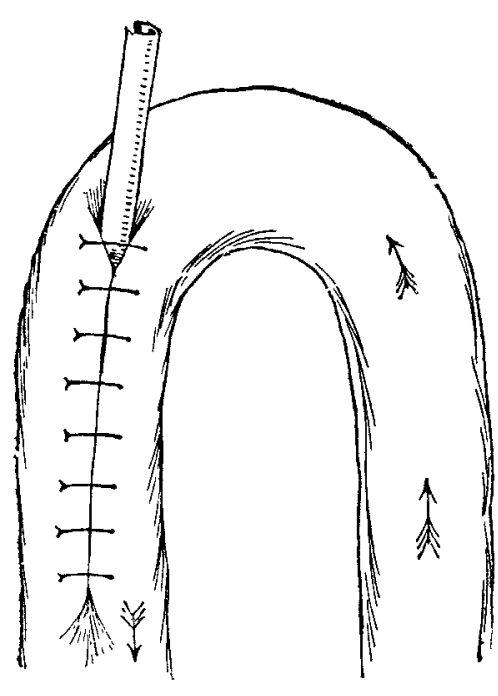

Second stage of Fig. 1 .

FIG. 3.

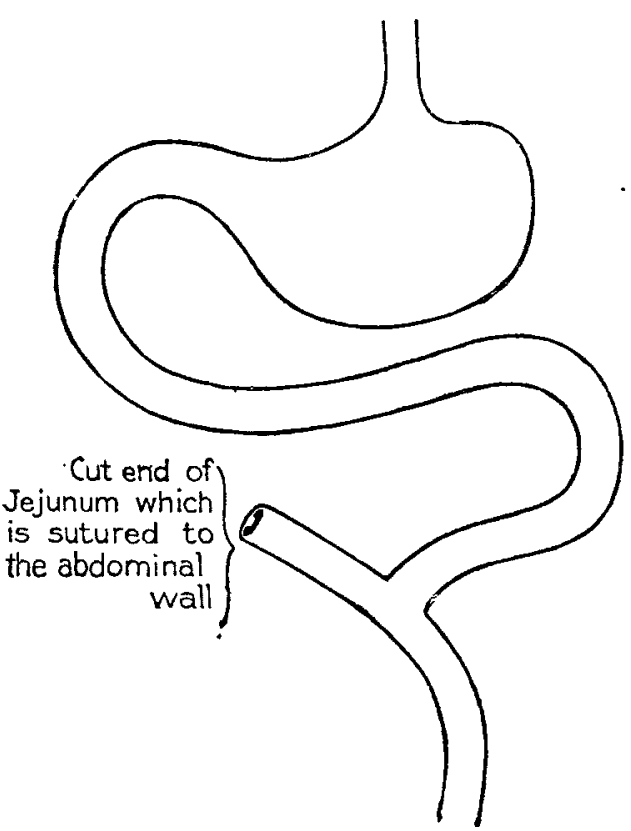

Jejunostomy by Maydl's method.

inserted and passed for three inches down the distal an of the loop; this is fixed to the margin of the incisio in the gut by a silk or Pagenstecher's suture and th entrance of the tube into the bowel is further guarde 
by two purse-string sutures, one over the other. The top of the loop is fixed to the skin by one or two stitches and the wound is closed. The patient can then be fed at once with some peptonised milk and brandy. The whole operation can be done in from 15 to 20 minutes and with very little visceral exposure (see Fig. 4). Should the patient

FIG. 4.

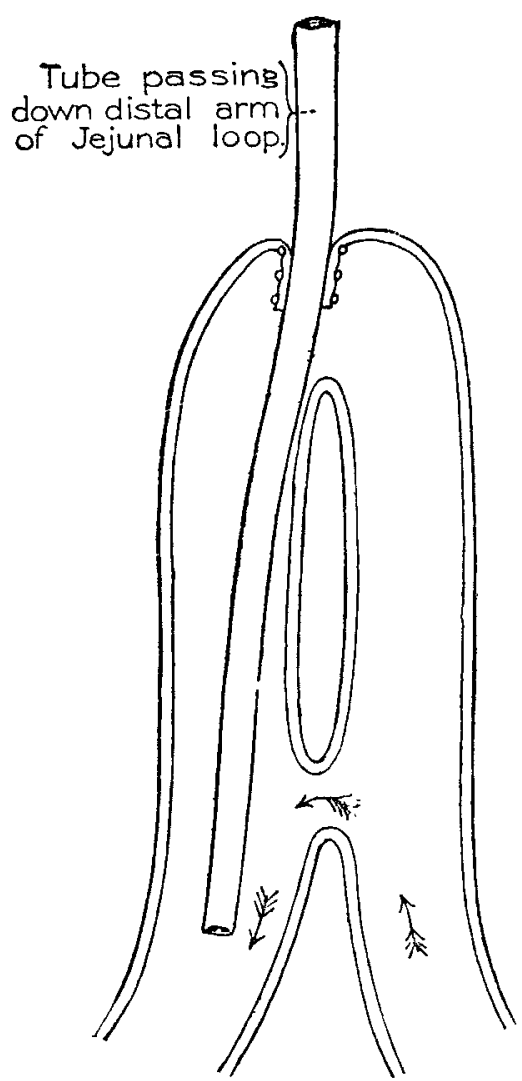

Jejunostomy by the author's method, now described.

be too ill to bear the little extra time occupied by the shortcircuiting the tube may be inserted as directed and surrounded by two or three purse-string sutures, a proceeding which can be accomplished in a few minutes. In this case the loop of bowel must not be brought to the skin but had better be fixed by sutures to the peritoneal margin and the aponeurosis in order to leave part of the lumen of the attached loop within the abdomen for the direct passage onwards of the intestinal fluid with the bile and pancreatic secretion. The following case affords an example of the relief that may follow this operation.

The patient, a woman, aged 46 years, was sent to see me on April 8th, 1904, when she was suffering great pain, which came on at frequent intervals, and she was vomiting five or six times a day; in fact, whenever she took food it was shortly afterwards vomited, the vomit being at times coffeeground in character. A tumour in the epigastrium of about the size of a large flat orange could be readily felt and at short intervals the whole stomach became hard and rigid. No enlarged glands could be felt in the groin or above the clavicle. There was no tenderness on pressure and the hard nodular tumour was suggestive of cancer. Though she gave a history of indigestion and loss of health for eight years or even longer the acute symptoms had only existed for six months.

On April 13th the abdomen was opened by a vertical incision through the inner margin of the right rectus, when the stomach was found to be involved in cancer from end to end, and as the glands along the lesser curvature were involved and others could be felt passing up through the opening in the diaphragm and a number also in the great omentum, it was clearly impossible to perform gastrectomy and impracticable to do a gastro-enterostomy. A loop of jejunum was therefore brought up and short-circuited by suture over a decalcified bone bobbin and a No. 12 Jaques catheter was inserted as just described. The wound was rapidly closed and the patient was put to bed in good condition, the whole operation only having occupied half an hour or less. A meal of peptonised milk was given at once and repeated every two hours. From the time of operation up to now the vomiting has never been repeated and she has completely lost her pain. On April 28th, 15 days after operation, I wrote to Dr. — : "Mrs. —_ has made a good recovery and is now able to take a little food by the mouth, though the feeding is chiefly by the tube. She is free from pain and the tumour is less." The patient returned home on the nineteenth day, having gained flesh and strength. After the patient's return home, on May 17th, I received a reply from Dr. - - : "Mrs. $\longrightarrow$ is doing wonderfully well. No pain or sickness, and taking as much food as she requires. She is very content and is, I think, gaining flesh and strength, so that the result is even better than you had hoped for and much better than I ever expected. She is very grateful." On Oct. 10th I had a letter from another practitioner to say that $\mathrm{Mrs}$. - had come under his care in North Devon and that she had expressed herself as much improved, though there was a tumour manifest. The patient survived for a year; ascites and secondary growths developed.

The case is of interest, not only from the relief afforded by jejunostomy but also as showing the sequence of cancer on ulcer-a not uncommon incidence-and one which might be prevented by timely intervention in the pre-cancerous stage. It is now clearly proved that ulcer of the stomach is a much more serious disease than was once thought and that many cases fail to yield to medical treatment, but I do not think that it is fully recognised how frequertly cancer follows on ulcer. In no less than $59 \cdot 3$ per cent. of the cases of cancer of the stomach on which I have operated the history, as in this case, clearly points to antecedent ulcer, the cancer being a later manifestation.

The operation of jejunostomy, which has a mortality of from 10 to 20 per cent., should be very seldom called for and would not be if the early diagnosis and treatment of gastric cancer were insisted on; but it must be distinctly recognised that if we intend to do the best for these cases we must make the diagnosis before tumour or coffee-ground vomiting leaves no doubt as to the nature of the ailment. Whenever a patient at or after middle age complains somewhat suddenly of indefinite gastric uneasines, pain, and vomiting, followed by progressive loss of weight and energy, associated with anæmia, the possibility of cancer of the stomach should be recognised and if no improvement takes place in two or three weeks an exploratory operation is more than justified. Our diagnosis can only be rendered certain by a digital examination, which may be effected through a small incision that can if needful be made under cocaine anæsthesia with little risk. Let us not forget that the early recognition of cancer of the stomach means not only the probability of relief but the possibility of cure in a certain proportion of cases.

Park-crescent, $\mathrm{W}$.

\section{THE SECOND DENTITION: ITS MEDICAL ASPECTS.}

\section{By HUBERT ARMSTRONG, M.D., Ch.B. Vict., M.D. LIVERP.,}

ASSISTANT PHYSICIAN, LIVERPOOL INFIRMARY FOR CHILDREN.

WHEN it is remembered that calcification begins in the case of the first permanent molar during the twenty-fifth week of embryonic life, and that the final eruption of the "wisdom tooth," the last of the permanent set to appear, does not take place till early adulthood, and when in connexion with the lengthy intervening period the importance of a sound dentition to the general health is considered, and vice versa the necessity of good health in the insurance of a sound dentition, it is obvious that the interest in the second dentition is not confined to the dental surgeon but concerns very largely the medical profession generally. It is not necessary here to enter into a description of the normal permanent teeth nor of the dates and the manner of their eruption, matters which are familiar to all of us. A passing reference, however, may be made to the first molar appearing at the sixth to seventh year. A friend informs me that the extraction of this tooth for caries and alveolar abscess was the most frequent operation which he was called upon to perform among some hundreds of youths in a naval training school to which be was formerly surgeon-dentist. This emphasises the need of a periodic examination for any

1 A paper read before the Liverpool Medical Institution on April 27th, 